\title{
THE JACOBIANS OF NON-MAXIMAL DEGREE
}

\author{
Sylwia Lara-Dziembek, Grzegorz Biernat, Edyta Pawlak \\ Institute of Mathematics, Czestochowa University of Technology \\ Częstochowa, Poland \\ sylwia.lara@im.pcz.pl,grzegorz.biernat@im.pcz.pl,edyta.pawlak@im.pcz.pl \\ Received: 30 October 2016; accepted: 15 November 2016
}

\begin{abstract}
In the article the leading forms of the polynomial mapping having the Jacobians of non-maximal degree are considered. In particular, the mappings having two zeros at infinity are discussed.
\end{abstract}

Keywords: jacobian, zeros at infinity

\section{Introduction}

The article presents the decomposition of leading forms of the polynomial mapping of two complex variables in the case when the Jacobian of this mapping does not meet maximal degree. In particular, the structure of these forms interest us in the case where the mapping has two zeros at infinity.

\section{The Jacobian having non - generic degrees}

Let $f_{m}, h_{n}$ be the forms of variables $X, Y$ of degrees $m, n$ respectively with the condition $m>n \geq 2$. Suppose that the Jacobian of forms $f_{m}, h_{n}$ vanish and represent the structures of these forms.

Lemma. Let $\operatorname{Jac}\left(f_{m}, h_{n}\right)=0$. Therefore

$$
f_{m}=a\left[\left(\alpha_{1} X+\beta_{1} Y\right)^{p_{1}} \ldots\left(\alpha_{k} X+\beta_{k} Y\right)^{p_{k}}\right]^{\tilde{m}}
$$

and

$$
h_{n}=b\left[\left(\alpha_{1} X+\beta_{1} Y\right)^{p_{1}} \ldots\left(\alpha_{k} X+\beta_{k} Y\right)^{p_{k}}\right]^{\tilde{n}}
$$


where:

$\tilde{m}=m /(m, n), \quad \tilde{n}=n /(m, n) \quad$ and $\quad p_{1}+\ldots+p_{k}=(m, n), \quad a, b, \alpha_{i}, \beta_{i} \in C$, $\operatorname{det}\left[\begin{array}{cc}\alpha_{i} & \beta_{i} \\ \alpha_{j} & \beta_{j}\end{array}\right] \neq 0$ for $i \neq j$, where $(m, n)$ means the greatest common divisor of the numbers $m$ and $n$.

Proof.

According to the Euler formula [1] we have

$$
X \frac{\partial f_{m}}{\partial X}+Y \frac{\partial f_{m}}{\partial Y}=m f_{m} \quad \text { and } \quad X \frac{\partial h_{n}}{\partial X}+Y \frac{\partial h_{n}}{\partial Y}=n h_{n}
$$

so using the Cramer rule we obtain

$$
X \operatorname{Jac}\left(f_{m}, h_{n}\right)=\operatorname{det}\left[\begin{array}{cc}
m f_{m} & \frac{\partial f_{m}}{\partial Y} \\
n h_{n} & \frac{\partial h_{n}}{\partial Y}
\end{array}\right] \text { and } Y \operatorname{Jac}\left(f_{m}, h_{n}\right)=\operatorname{det}\left[\begin{array}{cc}
\frac{\partial f_{m}}{\partial X} & m f_{m} \\
\frac{\partial h_{n}}{\partial X} & n h_{n}
\end{array}\right]
$$

Because $\operatorname{Jac}\left(f_{m}, h_{n}\right)=0$, then

$$
n \frac{\partial f_{m}}{\partial X} h_{n}=m \frac{\partial h_{n}}{\partial X} f_{m} \quad \text { and } \quad n \frac{\partial f_{m}}{\partial Y} h_{n}=m \frac{\partial h_{n}}{\partial Y} f_{m}
$$

so after dividing by the greatest common divisor $(m, n)$ of the numbers $m$ and $n$ we receive

$$
\tilde{n} \frac{\frac{\partial f_{m}}{\partial X}}{f_{m}}=\tilde{m} \frac{\frac{\partial h_{n}}{\partial X}}{h_{n}} \quad \text { and } \quad \tilde{n} \frac{\frac{\partial f_{m}}{\partial Y}}{f_{m}}=\tilde{m} \frac{\frac{\partial h_{n}}{\partial Y}}{h_{n}}
$$

This means that the logarithmic derivatives of the form $f_{m}, h_{n}$ respectively, satisfy equalities

$$
\left(\log f_{m}^{\tilde{n}}\right)_{X}^{\prime}=\left(\log h_{n}^{\tilde{m}}\right)_{X}^{\prime} \text { and }\left(\log f_{m}^{\tilde{n}}\right)_{Y}^{\prime}=\left(\log h_{n}^{\tilde{m}}\right)_{Y}^{\prime}
$$

therefore

$$
f_{m}^{\tilde{n}}=c h_{n}^{\tilde{m}}
$$

Thus the forms $f_{m}, h_{n}$ have the same factors. 
So

$$
f_{m}=a\left(\alpha_{1} X+\beta_{1} Y\right)^{\tilde{p}_{1}} \ldots\left(\alpha_{k} X+\beta_{k} Y\right)^{\tilde{p}_{k}}, \quad \tilde{p}_{1}+\ldots+\tilde{p}_{k}=m
$$

and

$$
h_{n}=b\left(\alpha_{1} X+\beta_{1} Y\right)^{\tilde{q}_{1}} \ldots\left(\alpha_{k} X+\beta_{k} Y\right)^{\tilde{q}_{k}}, \tilde{q}_{1}+\ldots+\tilde{q}_{k}=n
$$

According to formula (9) the exponents $\tilde{p}_{1}, \ldots, \tilde{p}_{k}, \tilde{q}_{1}, \ldots, \tilde{q}_{k}$ satisfy the equalities

$$
\tilde{p}_{1} \tilde{n}=\tilde{q}_{1} \tilde{m}, \ldots, \tilde{p}_{k} \tilde{n}=\tilde{q}_{k} \tilde{m}
$$

Since the numbers $\tilde{m}, \tilde{n}$ are relatively prime numbers, then

$$
\tilde{m}\left|\tilde{p}_{1}, \ldots, \tilde{m}\right| \tilde{p}_{k} \quad \text { and } \quad \tilde{n}\left|\tilde{q}_{1}, \ldots, \tilde{n}\right| \tilde{q}_{k}
$$

So

$$
\tilde{p}_{1}=p_{1} \tilde{m}, \ldots, \tilde{p}_{k}=p_{k} \tilde{m} \quad \text { and } \quad \tilde{q}_{1}=q_{1} \tilde{n}, \ldots, \tilde{q}_{k}=q_{k} \tilde{n}
$$

therefore

$$
m=\left(p_{1}+\ldots+p_{k}\right) \tilde{m} \quad \text { and } \quad n=\left(q_{1}+\ldots+q_{k}\right) \tilde{n}
$$

and at the same time the equality $p_{1}+\ldots+p_{k}=q_{1}+\ldots+q_{k}=(m, n)$ hold.

This means that

$$
f_{m}=a\left[\left(\alpha_{1} X+\beta_{1} Y\right)^{p_{1}} \ldots\left(\alpha_{k} X+\beta_{k} Y\right)^{p_{k}}\right]^{\tilde{m}}
$$

and

$$
h_{n}=b\left[\left(\alpha_{1} X+\beta_{1} Y\right)^{q_{1}} \ldots\left(\alpha_{k} X+\beta_{k} Y\right)^{q_{k}}\right]^{\tilde{n}}
$$

Assume

$$
\begin{aligned}
& f_{p}=\left(\alpha_{1} X+\beta_{1} Y\right)^{p_{1}} \ldots\left(\alpha_{k} X+\beta_{k} Y\right)^{p_{k}} \\
& h_{p}=\left(\alpha_{1} X+\beta_{1} Y\right)^{q_{1}} \ldots\left(\alpha_{k} X+\beta_{k} Y\right)^{q_{k}}
\end{aligned}
$$

where $p=p_{1}+\ldots+p_{k}=q_{1}+\ldots+q_{k}$. 
Therefore

$$
f_{m}=a f_{p}^{\tilde{m}} \text { and } \quad h_{n}=b h_{p}^{\tilde{n}}
$$

so

$$
\operatorname{Jac}\left(f_{m}, h_{n}\right)=a b \tilde{m} \tilde{n} f_{p}^{\tilde{m}-1} h_{p}^{\tilde{n}-1} \operatorname{Jac}\left(f_{p}, h_{p}\right)
$$

Thus $\operatorname{Jac}\left(f_{m}, h_{n}\right)=0$ exactly when $\operatorname{Jac}\left(f_{p}, h_{p}\right)=0$. However

$$
X \frac{\partial f_{p}}{\partial X}+Y \frac{\partial f_{p}}{\partial Y}=p f_{p} \quad \text { and } \quad X \frac{\partial h_{p}}{\partial X}+Y \frac{\partial h_{p}}{\partial Y}=p h_{p}
$$

and

$$
X \operatorname{Jac}\left(f_{p}, h_{p}\right)=p \operatorname{det}\left[\begin{array}{ll}
f_{p} & \frac{\partial f_{p}}{\partial Y} \\
h_{p} & \frac{\partial h_{p}}{\partial Y}
\end{array}\right] \text { and } Y \operatorname{Jac}\left(f_{p}, h_{p}\right)=p \operatorname{det}\left[\begin{array}{ll}
\frac{\partial f_{p}}{\partial X} & f_{p} \\
\frac{\partial h_{p}}{\partial X} & h_{p}
\end{array}\right]
$$

Then

$$
\frac{\partial f_{p}}{\partial X} h_{p}=\frac{\partial h_{p}}{\partial X} f_{p} \text { and } \frac{\partial f_{p}}{\partial Y} h_{p}=\frac{\partial h_{p}}{\partial Y} f_{p}
$$

so

$$
\frac{\frac{\partial f_{p}}{\partial X}}{f_{p}}=\frac{\frac{\partial h_{p}}{\partial X}}{h_{p}} \quad \text { and } \quad \frac{\frac{\partial f_{p}}{\partial Y}}{f_{p}}=\frac{\frac{\partial h_{p}}{\partial Y}}{h_{p}}
$$

This means that

$$
\left(\log f_{p}\right)_{X}^{\prime}=\left(\log h_{p}\right)_{X}^{\prime} \text { and }\left(\log f_{p}\right)_{Y}^{\prime}=\left(\log h_{p}\right)_{Y}^{\prime}
$$

therefore

$$
f_{p}=h_{p}=\left(\alpha_{1} X+\beta_{1} Y\right)^{p_{1}} \ldots\left(\alpha_{k} X+\beta_{k} Y\right)^{p_{k}}
$$

This completes the proof. 
Remark 1. Obviously we can assume that $p_{1} \geq \ldots \geq p_{k}$.

Corollary 1 [2]. Let $f=f_{m}+\tilde{f}, h=h_{n}+\tilde{h}$, where $\operatorname{det} \tilde{f}<m$, $\operatorname{det} \tilde{h}<n$. If $\operatorname{Jac}\left(f_{m}, h_{n}\right)=0$, then only zeros at infinity of the mapping $(f, h)$ are the factors of the form $f_{m}$ or $h_{n}$.

Corollary 2. If the numbers $m$ and $n$ are relatively prime and $\operatorname{Jac}\left(f_{m}, h_{n}\right)=0$, then $f_{m}=a(\alpha X+\beta Y)^{m}$ and $h_{n}=b(\alpha X+\beta Y)^{n}$. This means that the mapping $(f, h)$ has only one zero at infinity.

Corollary 3. Let $f=f_{m}+\tilde{f}, h=h_{n}+\tilde{h}$, where $\operatorname{det} \tilde{f}<m$ and $\operatorname{det} \tilde{h}<n$. Let $\operatorname{Jac}\left(f_{m}, h_{n}\right)=0$. If the mapping $(f, h)$ have two zeros at infinity, then

$$
f=\left(X^{k} Y^{l}\right)^{p}+\tilde{f} \text { and } h=\left(X^{k} Y^{l}\right)^{q}+\tilde{h}
$$

where $k \geq l, k$ and $l$ are relatively prime, $p>q \geq 1$.

Remark 2. In particular, can be consider the case $f=X^{k+1} Y^{k+1}+\tilde{f}$ and $h=X^{k} Y^{k}+\tilde{h}$ (we put $k=1=1, p=k+1, q=k$ ). The mappings of this type were considered in the paper [3].

\section{Conclusion}

The Jacobians of non-maximal degree appears for the mappings with the constant Jacobian. The Jacobians Conjecture [4-9] do not occur for non trivial classes of the mappings having the constant Jacobian and one or two zeros in infinity $[3,10]$. In the second case (two zeros in infinity) the leading forms of the mapping have the form given in the Corollary 2. The study of such mappings lead to the general classes of the mappings for which the Jacobian Conjecture does not take place.

\section{References}

[1] Mostowski A., Stark M., Elementy algebry wyższej, Wyd. Naukowe PWN, Warszawa 1997.

[2] Biernat G., The Jacobians of Lower Degree, Scientific Research of the Institute of Mathematics and Computer Science 2003, 2(1), 19-24.

[3] Lara-Dziembek S., Biernat G., Pawlak E., Woźniakowska M., A second example of non-Keller mapping, Journal of Applied Mathematics and Computational Mechanics 2016, 15(2), 65-70.

[4] Griffiths P., Harris J., Principles of Algebraic Geometry, New York 1978. 
[5] Mumford D., Algebraic Geometry I: Complex Projective Varieties, Springer Verlag, New York 1975.

[6] Shafarevich I.R., Basic Algebraic Geometry, Berlin, Springer-Verlag, New York 1974.

[7] Wright D., On the Jacobian conjecture, no. 3, 423-440, Illinois J. Math. 25, 1981.

[8] Van den Essen A., Polynomial automorphisms and the Jacobian conjecture, Progress in Matematics 190, Birkhäuser Verlag, Basel 2000.

[9] Bass H., Connell E.H., Wright D., The Jacobian conjecture: reduction of degree and formal expansion of the inverse, American Mathematical Society. Bulletin. New Series 1982, 7(2), 287-330.

[10] Pawlak E., Lara-Dziembek S., Biernat G., Woźniakowska M., An example of non-Keller mapping, Journal of Applied Mathematics and Computational Mechanics 2016, 15(1), 115-121. 